\title{
A Simple Approximation of an Infinite Dilution Solvating Energy of Ions in Dipolar and Polarizable Solvents
}

\author{
F. GRADZKI* \\ (Received October 3, 2008; in final form June 22, 2009)
}

\begin{abstract}
The infinite dilution solvating energy of ions in a solvent is considered. The ions are treated as hard spheres with point charges and the solvent molecules are treated as dipolar and polarizable hard spheres. The diameter of ion can be different than the diameter of solvent molecule. It can be seen that contribution from permanent dipolar moment of the solvent is bigger (and so more important) than the contribution from polarizability of the solvent.
\end{abstract}

PACS numbers: 31.70.Dk

\section{Introduction}

Experimental studies of ion-water clusters have provided insights into the microscopic aspects of hydration phenomena [1]. In this paper there is calculated the infinite dilution solvating energy of ions in a solvent of dipolar and polarizable hard spheres. The ions are treated as hard spheres with point charge $e$ and diameter $\sigma_{a a}$, and the solvent molecules are treated as dipolar and polarizable hard spheres with diameter $\sigma_{00}$.

Usually model calculations treat solvent molecules as dipolar hard spheres. It is interesting how much the results will be changed if we take into account also polarizability of solvent molecules. Also often in calculations diameter of ions is equal to diameter of solvent particles. In this paper we try to show how diameter of ions changes the results. Our results show that polarizability of solvent is also important. We also can see that solvating energy decreases with increasing diameter of ions.

\section{Theory}

Let us introduce the excess chemical potential $\mu^{\text {ex }}$ equal to [2]:

$$
\mu^{\mathrm{ex}}=\mu-\mu^{\mathrm{id}}
$$

where $\mu$ is the chemical potential of the ion in the solution and $\mu^{\mathrm{id}}$ is the chemical potential of the ideal gas. If we have one ion and $N$ solvent particles, then the energy of interaction can be written in the form

$$
\Phi_{1+N}=\Phi_{\mathrm{s}}(a, 1, \ldots, N)+\Phi_{\mathrm{e}}(a, 1, \ldots, N),
$$

where $\Phi_{\mathrm{s}}$ represents the short-ranged potential energy and $\Phi_{\mathrm{e}}$ represents the electrostatic long-ranged potential energy. The long-ranged potential energy is given by $[3,4]$ :

* 4015-7 $7^{\text {th }}$ Ave, \#7; Brooklyn, NY 11232, USA.

$$
\Phi_{\mathrm{e}}=-(1 / 2) \boldsymbol{M}^{+} \boldsymbol{T} \boldsymbol{M}-\boldsymbol{E}_{0}^{+} \boldsymbol{M}+(1 / 2) \boldsymbol{E}^{+} \boldsymbol{\alpha} \boldsymbol{E},
$$

where $\boldsymbol{M}, \boldsymbol{E}$ and $\boldsymbol{E}_{0}$ are $N \times 1$ column matrices with every element as an ordinary 3 -component vector. Similarly $\boldsymbol{T}$ and $\boldsymbol{\alpha}$ are $N \times N$ matrices with $\boldsymbol{T}_{i j}=\left(1-\delta_{i j}\right) \boldsymbol{T}\left(r_{i j}\right)$ and $\boldsymbol{\alpha}_{i j}=\boldsymbol{\alpha}(i) \delta_{i j}$ where $\delta_{i j}$ is the Kronecker $\delta$, the (.) indicates the transposed matrix. In particular $\boldsymbol{E}_{0 i}=$ $\boldsymbol{E}_{i a}=\boldsymbol{E}_{0}\left(\boldsymbol{r}_{i}\right)$ is the electric field at point $\boldsymbol{r}_{i}$ due to the charge of the ion. The $\boldsymbol{E}_{i a}$ and $\boldsymbol{T}\left(\boldsymbol{r}_{i j}\right)$ are defined by

$$
\begin{aligned}
& \boldsymbol{E}_{i a}=-e \nabla_{i}\left(r_{i a}^{-1}\right)=e r_{i a}^{-3} \boldsymbol{r}_{i a}=e r_{i a}^{-2} \boldsymbol{s}_{i a}, \\
& \boldsymbol{T}\left(\boldsymbol{r}_{i j}\right)=r_{i j}^{-3}\left(\boldsymbol{s}_{i j} \boldsymbol{s}_{i j}-\boldsymbol{I}\right),
\end{aligned}
$$

where $\boldsymbol{s}_{i j}=\left(\boldsymbol{r}_{i}-\boldsymbol{r}_{j}\right) / r_{i j}$ and $r_{i j}=\left|\boldsymbol{r}_{i j}\right|$, and $\boldsymbol{I}$ is the unit tensor. The matrices $\boldsymbol{M}$ of the actual dipole moments $\boldsymbol{M}_{i}$ and $\boldsymbol{E}$ of the microscopic electric fields $\boldsymbol{E}\left(\boldsymbol{r}_{i}\right)$ are related to the permanent dipole moments $\boldsymbol{m}_{i}=m \boldsymbol{n}_{i}$ ( $\boldsymbol{n}_{i}$ is unit vector), and values $\boldsymbol{E}_{0}\left(\boldsymbol{r}_{i}\right)$ of the electric field of ion by

$$
\begin{aligned}
& M=\boldsymbol{m}+\boldsymbol{\alpha} \boldsymbol{E}, \\
& \boldsymbol{E}=\boldsymbol{E}_{0}+\boldsymbol{T} \boldsymbol{M} .
\end{aligned}
$$

In order to eliminate $\boldsymbol{E}$ and $\boldsymbol{M}$, the inverse matrix $\boldsymbol{A}$ of $\boldsymbol{I}-\boldsymbol{\alpha} \boldsymbol{T}$ is introduced. It satisfies [3, 4]:

$$
\boldsymbol{A}-\boldsymbol{A} \alpha \boldsymbol{T}=\boldsymbol{I},
$$

where $\boldsymbol{I}_{i j}=\boldsymbol{I} \delta_{i j}$. Elimination of $\boldsymbol{E}$ and $\boldsymbol{M}$ by use of (6), (7) and (8) yields the final form

$$
\begin{aligned}
& \boldsymbol{M}=\boldsymbol{A}\left(\boldsymbol{m}+\boldsymbol{\alpha} \boldsymbol{E}_{0}\right), \\
& \boldsymbol{E}=\boldsymbol{A}\left(\boldsymbol{E}_{0}+\boldsymbol{T} \boldsymbol{m}\right) .
\end{aligned}
$$

Substituting Eq. (9) and Eq. (10) into Eq. (3) we obtain, after rearrangement, the electrostatic part of the potential energy in the form [5]:

$$
\begin{gathered}
\Phi_{\mathrm{e}}=-(1 / 2) \boldsymbol{m}^{+} \boldsymbol{T} \boldsymbol{A} \boldsymbol{m}-\boldsymbol{E}_{0}^{+} \boldsymbol{A} \boldsymbol{m} \\
-(1 / 2) \boldsymbol{E}_{0}^{+} \boldsymbol{A} \boldsymbol{\alpha} \boldsymbol{E}_{0} .
\end{gathered}
$$

For isotropic polarizability the $N \times N$ dimensional matrix $\boldsymbol{A}$ is equal to 


$$
\boldsymbol{A}=(\boldsymbol{I}-\alpha \boldsymbol{T})^{-1}=\sum(\alpha \boldsymbol{T})^{n},
$$

where sum is over $n \geq 0$. From Eq. (11) we see that

$$
\Phi_{\mathrm{e}}=\Phi_{0 \mathrm{e}}+\Phi_{1 \mathrm{e}}+\Phi_{2 \mathrm{e}},
$$

where

$$
\begin{aligned}
& \Phi_{0 \mathrm{e}}=-(1 / 2) \boldsymbol{m}^{+} \boldsymbol{T} \boldsymbol{A} \boldsymbol{m}, \\
& \Phi_{1 \mathrm{e}}=-\boldsymbol{E}_{0}^{+} \boldsymbol{A} \boldsymbol{m}, \\
& \Phi_{2 \mathrm{e}}=-(1 / 2) \boldsymbol{E}_{0}^{+} \boldsymbol{A} \boldsymbol{\alpha} \boldsymbol{E}_{0} .
\end{aligned}
$$

Introducing Eq. (12) into Eq. (15) and Eq. (16) we have

$$
\begin{aligned}
& \left\langle\Phi_{1 \mathrm{e}}\right\rangle=-\left\langle\boldsymbol{E}_{0}^{+} \boldsymbol{A} \boldsymbol{m}\right\rangle=-\left\langle\boldsymbol{E}_{0}^{+} \sum(\boldsymbol{\alpha} \boldsymbol{T})^{n} \boldsymbol{m}\right\rangle, \\
& \left\langle\Phi_{2 \mathrm{e}}\right\rangle=-(1 / 2)\left\langle\boldsymbol{E}_{0}^{+} \boldsymbol{A} \boldsymbol{\alpha} \boldsymbol{E}_{0}\right\rangle \\
& \quad=-(1 / 2)\left\langle\boldsymbol{E}_{0}^{+} \sum(\boldsymbol{\alpha} \boldsymbol{T})^{n} \boldsymbol{\alpha} \boldsymbol{E}_{0}\right\rangle
\end{aligned}
$$

where $\left\langle\Phi_{1 \mathrm{e}}\right\rangle$ denotes an average electrostatic energy of the one ion interacting with dipolar solvent particles, $\left\langle\Phi_{2 \mathrm{e}}\right\rangle$ denotes an average electrostatic energy of the one ion interacting with polarizable solvent particles.

Let us take only the first term in the sums. Then we have

$$
\begin{aligned}
& \left\langle\Phi_{1 \mathrm{e}}\right\rangle=-\int \boldsymbol{E}_{1 a} \cdot \boldsymbol{m}_{1} \eta^{(1)}\left(\boldsymbol{r}_{a}, 1\right) \mathrm{d}(1) \\
& \left\langle\Phi_{2 \mathrm{e}}\right\rangle=-(1 / 2) \alpha \int \boldsymbol{E}_{1 a} \cdot \boldsymbol{E}_{1 a} \eta^{(1)}\left(\boldsymbol{r}_{a}, 1\right) \mathrm{d}(1),
\end{aligned}
$$

where $\eta^{(1)}$ is the reduced distribution function in the solvent containing one ion [6]. It may be expressed by

$$
\eta^{(1)}\left(\boldsymbol{r}_{a}, 1\right)=n_{2}\left(\boldsymbol{r}_{a}, 1\right) / n_{1}^{a}\left(\boldsymbol{r}_{a}\right),
$$

where $n_{2}\left(\boldsymbol{r}_{a}, 1\right)$ is a two-particle distribution function. The contribution to $\left\langle\Phi_{1 \mathrm{e}}\right\rangle$ in Eq. (19) is $\mathrm{O}\left(\alpha^{0}\right)$, whereas the remaining terms in Eq. (17) are $\mathrm{O}(\alpha)$. In the asymptotic region of $\alpha \rightarrow 0$ these terms can be neglected.

Because we have

$$
n_{2}\left(\boldsymbol{r}_{a}, 1\right)=n_{1}^{a}(\rho / 4 \pi) g_{2}\left(\boldsymbol{r}_{a}, 1\right),
$$

where $g_{2}\left(\boldsymbol{r}_{a}, 1\right)$ is radial distribution function, then we obtain

$$
\begin{gathered}
\eta^{(1)}\left(\boldsymbol{r}_{a}, 1\right)=(\rho / 4 \pi) g_{2}\left(\boldsymbol{r}_{a}, 1\right) \\
=(\rho / 4 \pi)\left[1+h_{2}\left(\boldsymbol{r}_{a}, 1\right)\right],
\end{gathered}
$$

where $h_{2}\left(\boldsymbol{r}_{a}, 1\right)$ depends on orientation of dipolar solvent particle.

Then we have

$$
\begin{aligned}
& \left\langle\Phi_{1 \mathrm{e}}\right\rangle=-\rho /(4 \pi) \int \boldsymbol{E}_{1 a} \cdot \boldsymbol{m}_{1} h_{2}\left(\boldsymbol{r}_{a}, 1\right) \mathrm{d}(1) \\
& =-\rho /(4 \pi) \int \boldsymbol{E}_{1 a} \cdot \boldsymbol{m}_{1} h_{E}\left(\boldsymbol{r}_{a}, 1\right)\left(\boldsymbol{s}_{1 a} \cdot \boldsymbol{n}_{1}\right) \mathrm{d}(1) \\
& =-\rho m / 3 \int\left(\boldsymbol{E}_{1 a} \cdot \boldsymbol{s}_{1 a}\right) h_{E}\left(\boldsymbol{r}_{a}, 1\right) \mathrm{d} \boldsymbol{r}_{1 a} \\
& =-e \rho m / 3 \int r_{1 a}^{-2} h_{E}\left(\boldsymbol{r}_{a}, 1\right) \mathrm{d} \boldsymbol{r}_{1 a} \\
& =-4 \pi e \rho m / 3 \int h_{E}\left(r_{1 a}\right) \mathrm{d} r_{1 a},
\end{aligned}
$$

where $\boldsymbol{n}_{1}=\boldsymbol{m}_{1} / m_{1}, h_{\mathrm{E}}$ is the component of total correlation function for ion-dipole in the solvent of pure dipolar fluid and the integral in Eq. (24) is considered by Chan et al. [7]. The Fourier transform of $h_{\mathrm{E}}\left(r_{1 a}\right)$ satisfies the equation [7]:

$$
h_{E}(k)=c_{E}(k)+(1 / 3) \rho c^{+}(k) h_{E}(k),
$$

where for $c_{E}(k)$ and for $c^{+}(k)$ we used expressions given by Chan et al. [7], so we have from Eq. (25)

$$
h_{E}(k)=\left[1-(1 / 3) \rho c^{+}(k)\right]^{-1} c_{E}(k) \text {. }
$$

We take approximation $c^{+}(k)=c^{+}(0), \quad c_{E}(k)=$ $-4 \pi \mathrm{i} \beta e m / k$, then $h_{E}\left(\boldsymbol{r}_{a}, 1\right)$ is equal to

$$
h_{E}\left(r_{1 a}\right)=\beta e m\left[1-(1 / 3) \rho c^{+}(0)\right]^{-1} r_{1 a}^{-2} .
$$

Then the integral $\int h_{E}\left(r_{1 a}\right) \mathrm{d} r_{1 a}$ is equal to

$$
\int h_{E}\left(r_{1 a}\right) \mathrm{d} r_{1 a}=\left(\beta e m / \sigma_{1 a}\right)\left[1-(1 / 3) \rho c^{+}(0)\right]^{-1},
$$

where $\sigma_{1 a}$ is the smallest distance between the ion and a dipolar particle. It can be seen that [8]:

$$
1-(1 / 3) \rho c^{+}(0)=q(2 \xi) \equiv q_{+},
$$

where $q(\eta)$ is the Percus Yevick hard sphere inverse compressibility at reduced density $\eta$ :

$$
q(\eta)=(1+2 \eta)^{-2}(1-\eta)^{-4}
$$

and the constant $\xi$ is the solution of the equation

$$
q(2 \xi)-q(-\xi)=(4 \pi \rho / 3) \beta m^{2} \equiv 3 y .
$$

So we have

$$
\begin{aligned}
& \qquad h_{E}\left(\boldsymbol{r}_{a}, 1\right) \mathrm{d} r_{1 a}=\left(\beta e m / \sigma_{1 a}\right) / q_{+}, \\
& \text {and } \\
& \qquad\left\langle\Phi_{1 \mathrm{e}}\right\rangle=-(4 \pi / 3)\left(\mathrm{e}^{2} / \sigma_{1 a}\right) \beta m^{2} \rho / q_{+} .
\end{aligned}
$$

It can be seen that

$$
\begin{aligned}
& \left(4 \pi \beta m^{2} / 3\right) / q_{+}=3 y / q_{+}=\left(q_{+}-q_{-}\right) / q_{+} \\
& \quad=1-1 / \varepsilon_{1},
\end{aligned}
$$

where $\varepsilon_{1}$ is the pure dipolar solvent dielectric constant. So

$$
\left\langle\Phi_{1 \mathrm{e}}\right\rangle=-e^{2} / \sigma_{1 a}\left(1-1 / \varepsilon_{1}\right) .
$$

At infinite dilution, the Helmholtz free energy for charging a hard sphere in a dipolar solvent (at constant volume) can be obtained from $\left\langle\Phi_{1 \mathrm{e}}\right\rangle$ :

$$
\begin{gathered}
F_{1}(e)-F_{1}(0)=\int\left(\left\langle\Phi_{1 \mathrm{e}}\right\rangle / e\right) \mathrm{d} e \\
=-(1 / 2) \mathrm{e}^{2} / \sigma_{1 a}\left(1-1 / \varepsilon_{1}\right) .
\end{gathered}
$$

$F_{1}(q)$ is the Helmholtz free energy for transferring a hard sphere of charge $q$ from the gas phase to the dipolar solvent. Interaction of the hard sphere with $N$ dipolar solvent particles will be the same as interaction of the one hard sphere (with diameter of the ion) with $N$ other hard spheres with diameter of the dipolar particles (dipole moment of solvent particles does not interact with hard sphere). Then for the hard sphere in dipolar solvent we have [9]:

$$
\begin{aligned}
& \beta \mu_{0}^{\mathrm{ex}}=\left[-3+2(1-\eta)^{-1}+(1-\eta)^{-2}\right] \\
& \quad+2 \eta(1-\eta)^{-2}\left[1+(1-\eta)^{-1}\right] x^{3} \\
& \quad+(3 / 2)\left[1-(1-\eta)^{-2}\right](x-1)^{2}(x+1) \\
& \quad-(3 / 2)\left[1-(1-\eta)^{-1}\right]^{2} x(x-1)^{2}
\end{aligned}
$$




$$
\begin{aligned}
& +\left[-3 / 2+2(1-\eta)^{-1}-(1 / 2)(1-\eta)^{-2}\right. \\
& +\ln (1-\eta)]\left(3 x^{2}-2 x^{3}-1\right)
\end{aligned}
$$

where

$$
\begin{aligned}
& \eta=(\pi / 6)(N / V) \sigma_{00}^{3}, \\
& x=\sigma_{a a} / \sigma_{00} .
\end{aligned}
$$

The $\left\langle\Phi_{2 \mathrm{e}}\right\rangle$ is equal to

$$
\begin{aligned}
& \left\langle\Phi_{2 \mathrm{e}}\right\rangle=-(1 / 2) \alpha \rho /(4 \pi) \int \boldsymbol{E}_{1 a} \cdot \boldsymbol{E}_{1 a} g_{2}\left(\boldsymbol{r}_{a}, 1\right) \mathrm{d}(1) \\
& =-(1 / 2) \alpha \rho \int \boldsymbol{E}_{1 a} \cdot \boldsymbol{E}_{1 a} g_{2}\left(\boldsymbol{r}_{a}, 1\right) \mathrm{d} \boldsymbol{r}_{1 a} \\
& =-(1 / 2) \alpha \rho \mathrm{e}^{2} \int \boldsymbol{r}_{a 1}^{-4} g_{2}\left(\boldsymbol{r}_{a}, 1\right) \mathrm{d} \boldsymbol{r}_{1 a} \\
& =-2 \pi \alpha \rho \mathrm{e}^{2} \int r_{a 1}^{-2} g_{\mathrm{hs}}\left(r_{1 a}\right) \mathrm{d} r_{a 1},
\end{aligned}
$$

where the radial distribution function between ion and solvent particle is approximated by radial distribution function of hard spheres.

Then for an infinite dilution we have

$$
\begin{aligned}
\mu^{\mathrm{ex}} & =\mu_{0}^{\mathrm{ex}}+\mu_{1}^{\mathrm{ex}}+\mu_{2}^{\mathrm{ex}}=\mu_{0}^{\mathrm{ex}}-(1 / 2) \mathrm{e}^{2} / \sigma_{a 1}\left(1-1 / \varepsilon_{1}\right) \\
& -2 \pi \alpha \rho \mathrm{e}^{2} \int r_{1 a}^{-2} g_{\mathrm{hs}}\left(r_{1 a}\right) \mathrm{d} r_{1 a} .
\end{aligned}
$$

In the case when $g_{\mathrm{hs}}\left(r_{1 a}\right)=1$, we obtain

$$
\left\langle\Phi_{2 \mathrm{e}}\right\rangle=-(1 / 2) \mathrm{e}^{2} / \sigma_{1 a}\left(1-1 / \varepsilon_{2}\right)
$$

where $\varepsilon_{2}$ is dielectric constant of an ideal polarizable gas and is equal to $[10]$ :

$$
\varepsilon_{2}=(1-4 \pi \alpha \rho)^{-1},
$$

or

$$
\begin{aligned}
& \mu^{\mathrm{ex}}=\mu_{0}^{\mathrm{ex}}-(1 / 2) e^{2} / \sigma_{a 1}\left(1-1 / \varepsilon_{1}\right) \\
& -(1 / 2) e^{2} / \sigma_{a 1}\left(1-1 / \varepsilon_{2}\right) .
\end{aligned}
$$

The results for this approximation are presented in Table.

TABLE

The infinite dilution solvating energy of different sizes ions. The reduced density of solvent $\rho^{*}=0.7$, the reduced dipolar moment $m^{*}=2.0$ and the reduced scalar polarizability $\alpha^{*}=0.065$.

\begin{tabular}{c|c|c|c|c|c|c}
\hline \hline$x$ & $\beta \mu_{1}$ & $\left\langle\beta \Phi_{1 \mathrm{e}}\right\rangle$ & $\left\langle\beta \Phi_{2 \mathrm{e}}\right\rangle$ & $\beta F_{1}$ & $\beta \mu_{2}$ & $\beta \mu$ \\
\hline 0.5 & 2.35 & -260.83 & -76.24 & -130.42 & -206.65 & -204.29 \\
0.6 & 3.06 & -244.53 & -71.47 & -122.26 & -193.74 & -190.68 \\
0.7 & 3.90 & -230.15 & -67.27 & -115.07 & -182.34 & -178.44 \\
0.8 & 4.88 & -217.36 & -63.53 & -108.68 & -172.21 & -167.32 \\
0.9 & 6.03 & -205.92 & -60.19 & -102.96 & -163.15 & -157.11 \\
1.0 & 7.35 & -195.62 & -57.18 & -97.81 & -154.99 & -147.63 \\
1.1 & 8.86 & -186.31 & -54.45 & -93.15 & -147.61 & -138.74 \\
1.2 & 10.57 & -177.84 & -51.98 & -88.92 & -140.90 & -130.33 \\
1.3 & 12.49 & -170.11 & -49.72 & -85.05 & -134.77 & -122.28 \\
1.4 & 14.62 & -163.02 & -47.65 & -81.51 & -129.16 & -114.53 \\
1.5 & 17.00 & -156.50 & -45.74 & -78.25 & -123.99 & -106.99 \\
\hline
\end{tabular}

\section{Discussion}

We want to show that polarizability of water is giving important contribution (about $1 / 3$ ) to our results. Also we observe important decrease of solvating energy with increasing diameter of ions. We also can see that short-ranged contribution is small $\left(\right.$ see $\left.\beta \mu_{0}\right)$ and little be increasing with increase of diameter of ion.

Let us define a reduced density $\rho^{*}=\rho \sigma_{00}^{3}$ (where $\sigma_{00}$ is the diameter of dipolar and polarizable hard sphere), a reduced dipolar moment $m^{* 2}=\beta m^{2} / \sigma_{00}^{3}$, and at the beginning we assume that diameter of ion is the same as diameter of dipole particle, then we define a reduced charge $e^{* 2}=\beta e^{2} / \sigma_{00}$ and a scalar polarizability $\boldsymbol{\alpha}^{*}=\boldsymbol{\alpha} / \sigma_{00}^{3}$. The calculations were done for $\rho^{*}=0.7, m^{* 2}=4.0$, and $e^{* 2}=200.0$. This reduced charge is such that $T=298 \mathrm{~K}$, the ion is singly charged and $\sigma_{00}=2.8 \mathrm{~A}$. Patey and Valleau [11] took $\sigma_{00}=3.0 \mathrm{~A}$ and got $e^{* 2}=188.0$. Usually in literature $\sigma_{00}<3.0 \mathrm{~A}$, for example Robinson and Stokes [12] are giving $\sigma_{00}=2.8 \mathrm{~A}$.

At the above reduced density and reduced dipole moment we obtain from Eq. (31) value of $\xi=0.15712$, $K=0.42867, q_{+}=11.99$ and $\varepsilon_{1}=45.7$.

The average electrostatic energy of the one ion interacting with dipolar solvent particles given by Eq. (35) is in agreement with results of Garisto et al. [13].

The calculations have been done for ion diameter from $0.5 \sigma_{00}$ to $1.5 \sigma_{00}$. In Table there are given the values of the $\beta \mu_{0}^{\mathrm{ex}}$, the values of the Helmholtz free energy $F_{1}$, which are independent of polarizability, the values of the $\left\langle\beta \Phi_{2 \mathrm{e}}\right\rangle$ for $\alpha^{*}=0.065$, which are proportional to polarizability, the values of the $\beta \mu_{1}^{\mathrm{ex}}+\beta \mu_{2}^{\mathrm{ex}}$, and the values of the $\beta \mu^{\mathrm{ex}}$. First we can see that result depends in significant way on diameter of the ion.

One common view is that extending experimental studies to larger cluster sizes would give the single-ion absolute hydration free energies not obtainable by classical thermodynamic methods [1]. It seems that our paper shows classical calculations, which are giving first simple estimation of this problem. These calculations can be improved by taking terms with higher power of polarizability. Also we can see that the $\beta F_{1}$ are giving the main contribution in this approximation and that the contribution $\left\langle\beta \Phi_{2 \mathrm{e}}\right\rangle$ from the polarizability is important.

The polarizability of water is $\alpha=1.44 \mathrm{~A}^{3}$ [14], so is giving $\alpha^{*}=0.065$. Also we can make calculations for ions with smaller diameter than $0.5 \sigma_{00}$ and for ions with larger diameter than $1.5 \sigma_{00}$.

The diameter of ion $\mathrm{Na}^{+}$is about $0.7 \sigma_{00}$, the diameter of ion $\mathrm{Li}^{+}$is about $0.5 \sigma_{00}$, and the diameter of ion $\mathrm{OH}^{-}$ seems to be equal to $\sigma_{00}$ and the diameter of ion $\mathrm{Cl}^{-}$is about $1.3 \sigma_{00}$. We can try to compare our results with the results of Asthagiri et al. [1].

For $\mathrm{LiOH}$ the experimental value $\mu=-233.3 \mathrm{kcal} / \mathrm{mol}$ (see Table III in [1]) and from our simple approximation we have $\mu_{1}+\mu_{2}=-214.1 \mathrm{kcal} / \mathrm{mol}$, similarly for $\mathrm{NaOH}$ the experimental value $\mu=-208.1 \mathrm{kcal} / \mathrm{mol}$ and from our simple approximation we have $\mu_{1}+\mu_{2}=$ 
$-199.7 \mathrm{kcal} / \mathrm{mol}$. We see that differences between experiment and calculations of Asthagiri et al. [1], are of the same order as our calculations which are based on classical theory.

More attention should be given to the change in a solvent structure from the pure solvent to that involved in the solution around the solute [15]. A neutron diffraction experiments are combined with data of the Monte Carlo simulation [16-18], which are giving a number of solvent particles around ions. Molecular dynamics simulation has been performed to study the effect of the polarizabilities of model anions on the ionic solvation in water clusters [19]. The hydrations of $\mathrm{Na}^{+}$and $\mathrm{K}^{+}$were investigated by means of Monte Carlo simulation [20]. Many-body potentials for aqueous $\mathrm{Li}^{+}, \mathrm{Na}^{+}, \mathrm{Mg}^{2+}$, and $\mathrm{Al}^{3+}$ ions have been constructed [21].

The electrical field around the ions is very strong, so we can expect that solvent particles will be very close to ions. The number of solvent particles around one ion is limited by geometry. The maximal number of solvent particles in the first shell cannot be bigger than

$$
N_{1}=4 \pi\left(\sigma_{a 0}\right)^{2} /\left(\sigma_{00}\right)^{2},
$$

where $\sigma_{a 0}=\left(\sigma_{a a}+\sigma_{00}\right) / 2$.

More exactly the hydration numbers in the first shell can be calculated from the integral [22]:

$$
N_{1}=\rho \int g(r) 4 \pi r^{2} \mathrm{~d} r
$$

where integral is from $\sigma_{a 0}$ to the outer radius of the hydration shell. We usually do not know the $g(r)$ and the outer radius of the hydration shell, so for the first estimation we can use Eq. (45). For ions of $\mathrm{Na}^{+}$from Eq. (45) the $N_{1}$ is about 9 and for ions of $\mathrm{Li}^{+}$the $N_{1}$ is about 7 . In the literature for ions of $\mathrm{Na}^{+}[20,21]$ the $N_{1}$ is about 6 and for ions of $\mathrm{Li}^{+}$the $N_{1}$ is about 4 , which also should be given by Eq. (46).

\section{References}

[1] D. Asthagiri, L.R. Pratt, H.S. Ashbaugh, J. Chem. Phys. 119, 2702 (2003).

[2] H.L. Friedman, A Course in Statistical Mechanics, Prentice-Hall, Englewood Cliffs 1985.

[3] J. Stecki, Adv. Chem. Phys. 6, 413 (1962).
[4] M.S. Wertheim, Mol. Phys. 26, 1425 (1973).

[5] A. Bellemans, J. Stecki, Bull. Acad. Pol. Sci. Ser. Sci. Chim. 9, 343 (1961).

[6] J. Stecki, Bull. Acad. Pol. Sci. Ser. Sci. Chim. 9, 429 (1961).

[7] D.Y.C. Chan, D.J. Mitchell, B.W. Ninham, J. Chem. Phys. 70, 2946 (1979).

[8] M.S. Wertheim, J. Chem. Phys. 55, 4291 (1971).

[9] F. Gradzki, Chem. Phys. 130, 167 (1989).

[10] F. Gradzki, Acta Phys. Pol. A 61, 405 (1982).

[11] G.N. Patey, J.P. Valleau, J. Chem. Phys. 63, 2334 (1975).

[12] R.A. Robinson, R.H. Stokes, Electrolyte Solutions, 2nd rev. ed., Dover, Mineola (NY) 2002.

[13] F. Garisto, P.G. Kusalik, G.N. Patey, J. Chem. Phys. 79, 6294 (1983).

[14] G. Lamoureux, A.D. Mackerell, Jr., B. Roux, J. Chem. Phys. 119, 5185 (2003).

[15] M. Robson Wright, An Introduction to Aqueous Electrolyte Solutions, Antony Rowe, Chippenham 2007.

[16] A. Botti, F. Bruni, S. Imberti, M.A. Ricci, J. Chem. Phys. 120, 10154 (2004).

[17] A. Botti, F. Bruni, S. Imberti, M.A. Ricci, J. Chem. Phys. 121, 7840 (2004).

[18] S. Imberti, A. Botti, F. Bruni, G. Cappa, M.A. Ricci, A.K. Soper, J. Chem. Phys. 122, 194509 (2005).

[19] S. Yoo, Y.A. Lei, X.C. Zeng, J. Chem. Phys. 119, 6083 (2003).

[20] M. Carrillo-Tripp, H. Saint-Martin, I. Ortega-Blake, J. Chem. Phys. 118, 7062 (2003).

[21] D. Spangberg, K. Hermansson, J. Chem. Phys. 120, 4829 (2004).

[22] S. Koneshan, J.C. Rasaiah, J. Chem. Phys. 113, 8125 (2000). 